\title{
Etude des propriétés d'adsorption et de désorption du Plomb (Pb) et du Cadmium (Cd) par les sédiments d'une lagune tropicale en présence d'Allylthiourée
}

\author{
Marcellin Koffi YAO* et N'Guessan Louis-Berenger KOUASSI \\ Centre de Recherches Océanologiques (CRO), 29 rue des Pêcheurs, BP V 18 Abidjan, Côte d'Ivoire. \\ *Auteur Correspondant ; E-mail :ykmarco21@yahoo.fr ; markcelklindr@gmail.com
}

\section{RESUME}

Les métaux plomb $(\mathrm{Pb})$ et cadmium $(\mathrm{Cd})$ s'accumulent dans les réseaux trophiques aquatiques et peuvent présenter une menace pour la santé humaine et la faune. Une littérature relativement abondante existe sur la concentration totale de ces métaux dans les lagunes ivoiriennes. Cependant, aucune information n'est disponible pour prédire leur toxicité potentielle. Cette étude avait pour objectif de déterminer l'influence de la matière organique sur l'adsorption du plomb et du cadmium par les sédiments de la baie de Cocody. Les résultats ont montré que les grandes quantités d'allylthiourée (de matière organique) empêchent le cadmium de s'adsorber sur les sédiments aux concentrations de cadmium supérieures à $1 \mathrm{mg} / \mathrm{L}$, augmentant sa concentration dans la colonne d'eau. Aucune conclusion claire n'a été tirée pour le plomb. Les résultats ont aussi montré que les sédiments de la baie de Cocody sont très contaminés en cadmium et en plomb, mais que les fractions solubles à l'eau sont très faibles. Des études complémentaires d'adsorption-désorption prenant en compte des études cinétiques sur beaucoup plus d'échantillons de sédiments permettront de mieux comprendre le comportement et la toxicité des métaux dans la lagune Ebrié.

(C) 2015 International Formulae Group. All rights reserved.

Mots clés : Métaux traces, matière organique, adsorption, désorption, Estuaire.

\section{INTRODUCTION}

La contamination de l'atmosphère, de l'hydrosphère et des sédiments par les métaux lourds est aujourd'hui très préoccupant pour les pays développés et émergents. Les métaux lourds sont des éléments métalliques dont la densité est supérieure à 5 (Duffus, 2002). Ces métaux ne sont pas biodégradables et persistent donc dans l'environnement pendant de longues périodes (Morin et al., 2008; Bonanno and Giudice, 2010; Baralkiewick et al., 2010).

De plus, ils sont continuellement rejetés dans l'atmosphère, l'eau et les sédiments par diverses activités humaines. Cette accumulation des métaux lourds dans l'environnement peut se répercuter sur la santé des êtres humains et des animaux (Zheng et al., 2010 ; Sundaray et al, 2011). Les sédiments des milieux aquatiques agissent comme réservoirs ou puits de métaux et jouent par conséquent un rôle important dans la remobilisation des métaux.

L'accumulation des métaux dans les sédiments est gouvernée principalement par des processus physiques et chimiques dont l'adsorption et la désorption. La compréhension de ces processus d'adsorption- 
désorption est très importante pour la connaissance du cycle biogéochimique, de la biodisponibilité et de la toxicité des éléments métalliques dans le milieu aquatique. Il est bien connu que les conditions physicochimiques $\mathrm{du}$ milieu ( $\mathrm{pH}$, force ionique et potentiel d'oxydo-réduction) et la teneur en matière organique du milieu influencent les réactions d'adsorption-désorption (Hongyi et al., 2009; Chakraborty, 2010; Jho et al., 2011 ; Silva et al., 2011; Chakraborty et al., 2012). Il apparaît donc très important d'approfondir nos connaissances sur le rôle de la matière organique dans l'accumulation des métaux dans les sédiments. Kouassi et al. (2014), ont établi la distribution du cadmium dans les différentes phases des sédiments de la lagune Ebrié. Il a été révélé que la fraction de cadmium susceptible de se retrouver dans la colonne était largement supérieure à la moyenne de la croûte continentale terrestre. Cependant, cette étude ne prenait pas en compte l'interaction des éléments chimiques avec la matière organique sus-jacente.

Cette étude a pour objectif d'étudier les propriétés d'adsorption-désorption du plomb $(\mathrm{Pb})$ et du cadmium $(\mathrm{Cd})$ par les sédiments de la lagune Ebrié en présence d'allylthiourée.

\section{MATERIEL ET METHODES Echantillonnage}

Trois échantillons de sédiments de surface ont été prélevés dans la baie de Cocody (Figure 1) au moyen d'une petite benne de type Van-Veen, puis placés à l'obscurité dans une glacière $\left(4{ }^{\circ} \mathrm{C}\right)$. $\mathrm{Au}$ laboratoire, les sédiments ont été séchés à l'étuve à $30{ }^{\circ} \mathrm{C}$, tamisés sur $2 \mathrm{~mm}$, et conservés dans des flacons en plastique à l'obscurité et au frais $\left(20^{\circ} \mathrm{C}\right)$ pour analyses. La baie de Cocody a été choisie parce qu'elle est exposée aux rejets d'effluents de toutes sortes (domestiques, industriels, pharmaceutiques, etc.) sans traitement préalable. Trois stations ont été échantillonnées (Figure 1). La station 1 est proche d'une station de prétraitement d'eaux usées; la station 2 est située au centre de la baie. La station 3, localisée à l'extrémité de la baie reçoit les eaux usées urbaines des quartiers Cocody, 2 plateaux, Abobo, Adjamé, Williamsville, etc.
L'allylthiourée est un composé organique pour usage de laboratoire, d'analyse et de recherche. Le choix de cette molécule se justifie par la présence de groupements fonctionnels largement présents dans les milieux aquatiques et ayant une affinité pour les éléments métalliques.

\section{Protocole expérimental}

Une masse de $0,4 \mathrm{~g}$ de sédiments a été pesée dans des tubes en téflons de $50 \mathrm{~mL}$ préalablement lavés, rincés et séchés. Différents volumes de solutions de plomb ou de cadmium (1,6 à $20 \mathrm{~mL})$ de concentrations variant de 0,1 à $5 \mathrm{mg} / \mathrm{L}$ ont été préparés à partir de solutions standards de $1000 \mathrm{mg} / \mathrm{L}$ (MERCK, Allemagne) dans des tubes en téflon de $50 \mathrm{~mL}$. $1 \mathrm{~mL}$ d'Allylthiourée $98 \%$ (New jersey.usa:1-800-Acros-01) de concentration $(0,1$ ou $10 \mathrm{mg} / \mathrm{L})$ a été ajouté puis complété avec de l'eau distillée jusqu'à obtenir un volume final de $40 \mathrm{~mL}$. Le mélange obtenu a été agité pendant trois heures à l'aide d'un agitateur mécanique. Après décantation, une quantité de $10 \mathrm{~mL}$ du surnageant a été filtrée et conservée dans un réfrigérateur avant analyse.

\section{Méthode d'analyse}

La digestion des sédiments a été faite par la méthode de décomposition totale. Des échantillons de sédiments secs homogénéisés de $0,1 \mathrm{~g}$ ont été placés dans des tubes en téflon préalablement lavés à l'acide nitrique dilué et minéralisés à chaud, à l'aide de $1 \mathrm{~mL}$ d'eau régale $\left(\mathrm{HNO}_{3}: \mathrm{HCl} ; 1: 3\right.$, v/v) et $3 \mathrm{~mL}$ de $\mathrm{HF}$ concentré $48 \%$, pur. Le chauffage s'est fait à $120{ }^{\circ} \mathrm{C}$ sur réchaud jusqu'à digestion totale. Après refroidissement à l'air ambiant, les résidus sont repris dans une solution d'acide borique (2,8 g dans $20 \mathrm{~mL}$ d'eau distillée). Le volume final a été ramené à $50 \mathrm{~mL}$ et laissé au repos pendant toute une nuit.

Les concentrations en $\mathrm{Pb}$ et $\mathrm{Cd}$ des solutions obtenues ont été mesurées par spectrophotomètre d'absorption atomique (SAA) (SpectrAA100 Varian spectrophotometer, Japan) au four graphite. La limite détection a été de $5 \mathrm{ng} / \mathrm{L}$ pour $\mathrm{Cd}$ et $\mathrm{Pb}$. 


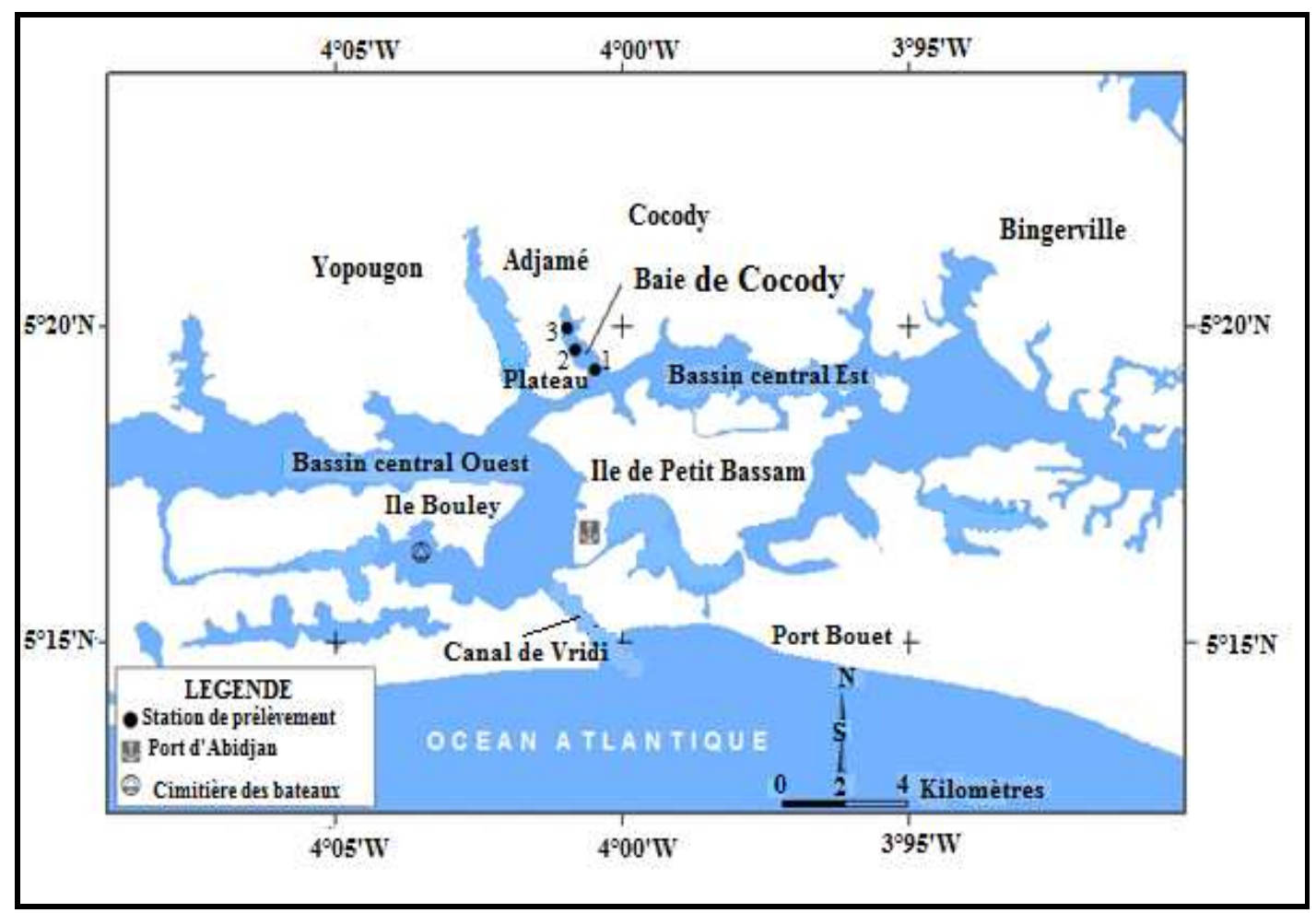

Figure 1 : Localisation des stations de prélèvement des sédiments dans la baie de Cocody.

\section{RESULTATS}

Concentrations totales du plomb et du cadmium des sédiments de la baie de Cocody

Les concentrations totales du plomb et du cadmium des sédiments échantillonnés en baie de Cocody sont reportées dans le Tableau 1. Il apparaît que les concentrations totales du plomb et du cadmium dans les sédiments de la baie de Cocody sont 8 fois plus élevées que les valeurs moyennes de la croûte continentale terrestre. Ces valeurs élevées pourraient s'expliquer par l'utilisation du plomb et du cadmium dans les différentes activités de l'homme (urbanisation, industrialisation, agriculture) dans l'agglomération Abidjan. Il importe de noter que les concentrations totales des éléments traces dans les sédiments de la baie de Cocody, y compris le plomb et le cadmium, ont fait l'objet d'une publication (Kouassi et al., 2015).

$\mathrm{Vu}$ que la concentration totale n'apporte aucune information sur la toxicité des métaux dans les sédiments, cette étude se focalise sur l'étude des propriétés d'adsorption-désorption du plomb et du cadmium dans la baie de Cocody.

Fraction disponible du plomb et du cadmium des sédiments de la baie de Cocody

Afin de déterminer la fraction disponible du plomb et du cadmium des sédiments de la baie de Cocody, nous avons déterminé leurs fractions solubles à l'eau par agitation mécanique pendant 8 heures. Nous avons obtenues des valeurs de $0,24 \mu \mathrm{g} / \mathrm{g}$ pour le plomb et de $0,12 \mu \mathrm{g} / \mathrm{g}$ pour le cadmium (Tableaux 2 et 3 ). Ces valeurs sont très 
faibles, comparées aux concentrations totales rapportées ci-dessous. Cependant, notons que la fraction soluble à l'eau ne représente qu'une partie de la fraction totale disponible. Une étude précédente prenant en compte la distribution du cadmium dans la fraction échangeable, la fraction liée aux carbonates et la fraction liée à la matière organique avait montré que la fraction totale susceptible de se retrouver dans la colonne d'eau en cas de changement des conditions redox des milieux se situe entre 33 et $50 \%$. Cette fraction est très élevée et indique qu'une grande partie de cadmium pourrait s'accumuler dans les organismes vivants dans la baie de Cocody.

\section{Influence de la matière organique (allylthiourée) sur l'adsorption-désorption des sédiments}

Afin de mieux comprendre l'influence de l'allylthiourée sur l'adsorption-désorption $\mathrm{du}$ plomb et du cadmium sur les sédiments, nous avons dans un premier temps mené l'étude de l'adsorption-désorption du plomb et du cadmium sur les sédiments en l'absence de l'allylthiourée. Les résultats sont présentés dans les Tableaux 2 et 3 . Nous observons que pour la solution initiale ne contenant pas de plomb ou de cadmium, il y a passage de plomb ou de cadmium des sédiments vers la solution à l'équilibre (Tableaux 2 et 3), indiquant une désorption. $\mathrm{Au}$ contraire, lorsque la concentration initiale en plomb et en cadmium de la solution initiale $(\mathrm{C} 0)$ varie entre 300 et $5000 \mu \mathrm{g} / \mathrm{L}$; Ce, qui représente la concentration restante des éléments plomb et cadmium dans la solution à l'équilibre, est plus petite $(0,82$ à $6,63 \mu \mathrm{g} / \mathrm{L}$ pour le plomb et 5,79 à $21,57 \mu \mathrm{g} / \mathrm{L}$ pour le cadmium) que $\mathrm{C} 0$. Cette perte de métal dans la solution initiale est attribuable à l'adsorption de métal par les sédiments. La représentation de l'isotherme d'adsorption $[\mathrm{Ce}=\mathrm{f}(\mathrm{Qe})]$ n'a donnée aucune tendance claire. Cependant, nous observons que la quantité de métal adsorbée augmente significativement avec la quantité initiale de métal dans la solution. Cette observation indique que soit les sédiments de la baie de Cocody possèdent une forte capacité d'adsorption du plomb et du cadmium, soit toute la quantité de métal perdue par la solution initiale n'a pas été adsorbée par le sédiment.

Dans un deuxième temps, nous avons mené l'étude de l'adsorption-désorption du plomb et du cadmium sur les sédiments en présence de l'allylthiourée. Les isothermes d'adsorption sont présentées dans les Tableaux 4 et 5. L'observation du Tableau 4 fait apparaître qu'aux faibles concentrations de cadmium dans la solution, une grande quantité d'allylthiourée est favorable à l'adsorption de cadmium par le sédiment. Au contraire, lorsque la concentration du cadmium est élevée dans le milieu ( $\geq 1 \mathrm{mg} / \mathrm{L})$, la quantité de cadmium adsorbée sur les sédiments diminue avec les grandes quantités d'allylthiourée, et donc de matière organique. Ceci indique que la capacité d'adsorption du cadmium par le sédiment décroît avec l'augmentation de la concentration d'allylthiourée dans la solution aux concentrations élevées de cadmium dans la solution.

En ce qui concerne le plomb, aucune tendance claire n'a été observée (Tableau 5). Une répétition de cette expérience sur plusieurs échantillons de sédiments conduira à une conclusion plus fiable. 
Tableau 1 : Concentrations totales du plomb et du cadmium du sédiment échantillonné en baie de Cocody.

\begin{tabular}{lcc}
\hline & $\mathbf{C d}(\boldsymbol{\mu g} / \mathbf{g})$ & $\mathbf{P b}(\boldsymbol{\mu} \mathbf{g} / \mathbf{g})$ \\
\hline moyenne & 0,77 & 99,77 \\
Ecart-type & $\pm 0,27$ & $\pm 12,50$ \\
Croûte continentale terrestre & 0,10 & 12 \\
\hline
\end{tabular}

Tableau 2 : Quantité de plomb adsorbé par gramme de sédiment.

\begin{tabular}{cccc}
\hline $\mathbf{C 0}(\boldsymbol{\mu g} / \mathbf{L})$ & $\mathbf{C e}(\boldsymbol{\mu g} / \mathbf{L})$ & $\mathbf{Q e}(\boldsymbol{\mu g} / \mathbf{g})$ & $\mathbf{Q e}^{\prime}(\boldsymbol{\mu g} / \mathbf{g})$ \\
\hline 0 & 2,48 & $-0,25$ & 0 \\
300 & 0,82 & 29,88 & 29,63 \\
500 & 11,27 & 48,80 & 48,55 \\
1000 & 3,23 & 99,60 & 99,35 \\
1500 & 10,70 & 148,63 & 148,39 \\
2000 & 1,56 & 199,45 & 199,20 \\
2500 & 8,12 & 248,88 & 248,63 \\
4000 & 2,04 & 399,20 & 398,95 \\
5000 & 5,87 & 498,67 & 498,42 \\
10000 & 8,55 & 996,90 & 996,65 \\
30000 & 6,65 & 2994,10 & 2993,85 \\
50000 & 6,63 & 4994,34 & 4994,09 \\
\hline
\end{tabular}

$\mathrm{CO}$ et Ce représentent la concentration initiale et à l'équilibre de plomb dans la solution surnageante. Qe est la quantité de plomb adsorbée par gramme de sédiments. Qe' est la quantité de plomb adsorbée par gramme de sédiments lorsque la quantité maximale de plomb susceptible d'être désorbée n'est pas prise en compte.

Tableau 3 : Quantité de cadmium adsorbé par gramme de sédiment.

\begin{tabular}{lccc}
\hline $\mathbf{C 0}(\boldsymbol{\mu g} / \mathbf{L})$ & $\mathbf{C e}(\boldsymbol{\mu g} / \mathbf{L})$ & $\mathbf{Q e}(\boldsymbol{\mu g} / \mathbf{g})$ & $\mathbf{Q e}^{\prime}(\boldsymbol{\mu g} / \mathbf{g})$ \\
\hline 0 & 1,19 & $-0,12$ & 0 \\
300 & 5,79 & 29,41 & 0,00 \\
500 & 11,17 & 48,82 & 48,55 \\
1000 & 10,69 & 98,78 & 99,35 \\
1500 & 8,98 & 149,03 & 148,39 \\
2000 & 13,90 & 198,31 & 199,20 \\
2500 & 10,28 & 248,47 & 248,63 \\
4000 & 10,99 & 398,70 & 398,95 \\
5000 & 14,22 & 498,20 & 498,42 \\
10000 & 16,69 & 997,58 & 996,65 \\
30000 & 20,77 & 2996,43 & 2993,85 \\
50000 & 21,57 & 4991,60 & 4994,09 \\
\hline
\end{tabular}

$\mathrm{CO}$ et Ce représentent la concentration initiale et à l'équilibre de cadmium dans la solution surnageante. Qe est la quantité de cadmium adsorbée par gramme de sédiments. Qe' est la quantité de cadmium adsorbée par gramme de sédiments lorsque la quantité maximale de cadmium susceptible d'être désorbée n'est pas prise en compte. 
Tableau 4 : Quantité de cadmium adsorbé par gramme de sédiment en présence d'allylthiourée à $10 \mathrm{mg} / \mathrm{L}$ et à $0,1 \mathrm{mg} / \mathrm{L}$.

\begin{tabular}{lccccc}
\hline $\mathbf{C 0}(\boldsymbol{\mu g} / \mathbf{L})$ & $\mathbf{C e} \mathbf{1 0}(\boldsymbol{\mu g} / \mathbf{L})$ & $\mathbf{C e} \mathbf{0 , 1}(\mathbf{m g} / \mathbf{L})$ & $\mathbf{Q e}^{\mathbf{1 0}}{ }^{\prime}(\boldsymbol{\mu g} / \mathbf{g})$ & $\mathbf{Q e}^{\prime} \mathbf{0 , 1}(\boldsymbol{\mu g} / \mathbf{g})$ & $\mathbf{Q e}^{\prime} \mathbf{1 0} / \mathbf{Q e}^{\prime} \mathbf{0 , 1}$ \\
\hline 0 & 1,19 & 5,76 & 0,00 & 0,00 & - \\
100 & 1,55 & 0,00 & 9,72 & 9,12 & 1,07 \\
200 & 2,68 & 0,00 & 19,60 & 19,00 & 1,03 \\
300 & 6,10 & 0,01 & 29,22 & 28,82 & 1,01 \\
400 & 3,34 & 0,00 & 39,47 & 38,46 & 1,03 \\
500 & 4,52 & 0,00 & 49,42 & 48,23 & 1,02 \\
700 & 3,24 & 0,00 & 69,49 & 68,71 & 1,01 \\
1000 & 8,93 & 0,01 & 98,79 & 98,50 & 1,00 \\
1500 & 9,45 & 0,01 & 148,64 & 148,45 & 1,00 \\
2000 & 11,82 & 0,01 & 198,35 & 197,81 & 1,00 \\
2500 & 12,67 & 0,01 & 248,37 & 247,97 & 1,00 \\
5000 & 17,10 & 0,02 & 497,18 & 497,31 & 1,00 \\
\hline
\end{tabular}

$\mathrm{C} 0$ et Ce représentent la concentration initiale et à l'équilibre de cadmium dans la solution surnageante. Qe' est la quantité de cadmium adsorbée par gramme de sédiments lorsque la quantité maximale de cadmium susceptible d'être désorbée n'est pas prise en compte.

Tableaux 5 : Quantité de plomb adsorbé par gramme de sédiment en présence d'allylthiourée à 10 $\mathrm{mg} / \mathrm{L}$ et à $0,1 \mathrm{mg} / \mathrm{L}$.

\begin{tabular}{cccccc}
\hline $\mathbf{C 0}(\boldsymbol{\mu g} / \mathbf{L})$ & $\mathbf{C e} \mathbf{1 0}(\boldsymbol{\mu g} / \mathbf{L})$ & $\mathbf{C e ~ 0 , 1}(\boldsymbol{\mu g} / \mathbf{L})$ & $\mathbf{Q e}^{\prime} \mathbf{1 0}(\boldsymbol{\mu g} / \mathbf{g})$ & $\mathbf{Q e}^{\prime} \mathbf{0 , 1}(\boldsymbol{\mu g} / \mathbf{g})$ & $\mathbf{Q e}^{\prime} \mathbf{1 0} / \mathbf{Q e}^{\mathbf{0}, \mathbf{1}}$ \\
\hline 0 & 1,21 & 0,41 & 0,00 & 0,00 & \\
100 & 1,42 & 3,65 & 9,72 & 9,50 & 1,02 \\
200 & 4,46 & 1,95 & 19,43 & 19,66 & 0,99 \\
300 & 1,54 & 2,43 & 29,69 & 29,60 & 1,00 \\
400 & 13,05 & 3,35 & 38,52 & 39,51 & 0,97 \\
500 & 0,38 & 2,50 & 49,82 & 49,52 & 1,01 \\
700 & 3,29 & 7,20 & 69,50 & 69,04 & 1,01 \\
1000 & 5,75 & 3,14 & 99,15 & 99,37 & 1,00 \\
1500 & 2,05 & 2,94 & 149,67 & 149,44 & 1,00 \\
2000 & 8,68 & 1,00 & 198,76 & 199,43 & 1,00 \\
2500 & 1,07 & 10,66 & 249,65 & 248,75 & 1,00 \\
5000 & 33,30 & 168,87 & 496,30 & 482,87 & 1,03 \\
\hline
\end{tabular}

$\mathrm{C} 0$ et Ce représentent la concentration initiale et à l'équilibre de plomb dans la solution surnageant. Qe' est la quantité de plomb adsorbée par gramme de sédiments lorsque la quantité maximale de plomb susceptible d'être désorbée n'est pas prise en compte. 


\section{DISCUSSION}

Les différentes phases susceptibles de favoriser l'adsorption des éléments métalliques par le sédiment sont la matière organique, les oxydes de fer et de manganèse, les argiles et les carbonates. Kouassi et al. (2015) ont montré dans une récente étude que les sédiments de la baie de Cocody sont très chargés en carbone organique total avec des concentrations variant entre 2 et $6 \mathrm{mg} / \mathrm{g}$ de sédiment. Dans cette même étude, les concentrations de $\mathrm{Cd}$ et de $\mathrm{Pb}$ ont été trouvées fortement corrélées à la concentration en carbone organique total des sédiments. Les fortes capacités d'adsorption du cadmium et du plomb enregistrées dans cette étude sont en grande partie imputables aux teneurs élevées des sédiments de la baie de Cocody en matière organique. En effet, la matière organique des sédiments, formée à partir de la décomposition des plantes et des débris animaux, contient des substances humiques qui, de par leurs nombreux groupes fonctionnels, peuvent fixer les ions métalliques par complexation (Chakraborty, 2010).

Dans la solution d'étude, l'allylthiourée et le sédiment sont les substrats d'adsorption des ions cadmium et plomb. Nos résultats ont montré qu'en l'absence d'allylthiourée, les sédiments de la baie de Cocody adsorbent plus de cadmium. La diminution de la quantité de cadmium adsorbée par le sédiment en présence de l'allylthiourée pourrait s'expliquer par le fait que l'allylthiourée possède plusieurs sites pour la complexation du cadmium. L'allylthiourée peut ainsi former des complexes cadmium-allylthiourée solubles et décroître la possibilité d'adsorption des métaux par le sédiment. La complexation se fera principalement par les fonctions thiol et thione de la molécule d'allylthiourée. La formation de complexes cadmiumallylthiourée est corroborée par Jalilehvand et al. (2012) qui ont montré que les molécules de thiourée, une molécule dont dérive l'allylthiourée, forment un mélange de complexes hydratés en solution aqueuse à la température ambiante, et que différents types de complexes prédominent selon la concentration en thiourée. La prédominance des différents types de complexes métalallylthiourée selon la concentration en allylthiourée, couplée à la supplantation de l'adsorption des ions cadmium par les molécules d'allylthiourée par rapport aux sites d'adsorption des sédiments pourraient expliquer la diminution de la capacité d'adsorption des sédiments aux concentrations élevées d'allylthiourée. Cependant, une adsorption d'allylthiourée par les sédiments augmenterait l'adsorption par l'augmentation des sites d'adsorption. Ceci pourrait expliquer le fait que les quantités de cadmium adsorbées par gramme de sédiments sont plus élevées pour des solutions contenant $10 \mathrm{mg} / \mathrm{L}$ d'allylthiourée par rapport à celles contenant $0,1 \mathrm{mg} / \mathrm{L}$ d'allylthiourée aux faibles concentrations de cadmium. D'autres facteurs tels que le $\mathrm{pH}$ et la force ionique pourraient influencer la désorption et l'adsorption de $\mathrm{Cd}$ et $\mathrm{Pb}$ par les sédiments. Notons que Chakraborty et al. (2014) ont observé dans une étude similaire que les acides humiques diminuaient la capacité d'adsorption de l'ion mercure (II) des sédiments collectés dans les estuaires de Visakhapatnam, Kakinada, Bhimli et de Gangavaram en Inde.

Ce résultat est très significatif du point de vu environnementale. En effet, le thiourée et ses composés dérivés sont largement répandus dans l'environnement. Leur présence dans le milieu aquatique favorisera donc la formation de complexes métalthiourée solubles, ce qui augmenterait la concentration des éléments traces dans la colonne d'eau, et par conséquent, leur biodisponibilité. Cependant, des études 
prenant en compte des échantillonnages passifs sont nécessaires pour confirmer ces hypothèses puisque ces complexes formés pourraient être défavorables à la bioaccumulation.

\section{Conclusion}

Cette étude nous a permis de comprendre l'influence de la matière organique sur l'adsorption du cadmium par les sédiments de baie de Cocody. L'étude montre que les grandes quantités d'allylthiourée (de matière organique) empêchent le cadmium de s'adsorber sur les sédiments aux concentrations de cadmium supérieures à 1 $\mathrm{mg} / \mathrm{L}$, augmentant sa concentration dans la colonne d'eau. Les résultats de cette étude ont aussi montré que les sédiments de la baie de Cocody sont très contaminés en cadmium et en plomb et que les fractions solubles à l'eau sont très faibles. Des études complémentaires d'adsorption-désorption prenant en compte des études cinétiques sur beaucoup plus d'échantillons de sédiments permettront de mieux comprendre le comportement et la toxicité des métaux dans la lagune Ebrié.

\section{REFERENCES}

Bonanno G, Giudice RL. 2010. Heavy metal bioaccumulation by the organs of Phragmites australis (common reed) and their potential use as contamination indicators. Ecol. Indic., 10: 639-645.

Braralkiewiz D, Hanć A, Gramowska,H. 2010. Simultaneous determination of $\mathrm{Cd}$, $\mathrm{Cr}, \mathrm{Cu}, \mathrm{Ni}, \mathrm{Pb}$ and $\mathrm{Zn}$ in sewage Sludge by Slurry introduction ICP-OES method. Intern. Environ. Anal. Chem., 90: 10251035.

Chakraborty P, 2010. Study of cadmiumhumic interactions and determination of stability constants of cadmium- humate complexes from their diffusion coefficients obtained by scanned stripping voltammetry and dynamic light scattering techniques. Anal. Chim. Acta, 659: 137143.

Chakraborty P, Brij MS, Raghunadh Babu PV, Koffi MY, Saranya J. 2014. Impact of total organic carbon (in sediments) and dissolved organic carbon (in overlying water column) on $\mathrm{Hg}$ sequestration by coastal sediments from the central east coast of India. Mar. Pollut. Bull., 79: 342347.

Chakraborty P, Raghunadh Babu PV, Sarma VV. 2012. A study of lead and cadmium speciation in some estuarine and coastal sediments. Chem. Geol., 294: 217-225.

Duffus JH. 2002. Heavy metals-A meaningless term? Pure App. Chem., 74(5): 793-807.

Hongyi N, Wenjing D, Qunke W, Xiengeng C. 2009. Potential toxic risk of the Pearl River in South China. J. Environ. Sci., 21: 1053-1058.

Jalilehvand F, Amini, Z, Parmar K. 2012. Cadmium (II) Complex Formation with Selenourea and Thiourea in Solution: An $\mathrm{XAS}$ and ${ }^{113} \mathrm{Cd}$ NMR Study. Inorg. Chem., 51: 10619-10630.

Jho EH, Lee SB, Kim YJ, Nam K. 2011. Facilitated desorption and stabilization of sediment-bound $\mathrm{Pb}$ and $\mathrm{Cd}$ in the presence of birnessite and apatie. $J$. Hazard. Mater., 188: 206-211.

Kouassi NLB, Yao KM, Trokourey A, Soro MB. 2015. Distribution, sources, and possible adverse biological effects of trace metals in surface sediments of a tropical estuary. Environ Forensics, 16. Doi : 10.1080/15275922.2014.991433.

Kouassi NLB, Yao KM, Trokourey A, Soro MB. 2014. Preliminary assessment of cadmium mobility in surface sediments of a tropical estuary. Bull. Chem. Soc. Ethiop., 28(2): 1-10. 
Morin S, Duong TT, Dabrin A, Coynel A, Herlory O, Baudrimont M, Delmas F, Durrieu G, Scha fer J, Winterton P, Blanc G, Coste M. 2008. Long-term survey of heavy metal pollution, biofilm contamination and diatom community structure in the Riou Mort Waterhed, South-West France. Environ. Pollut., 151: $532-542$.

Silva LFO, Izquierdo M, Querol X, Finkelman RB, Oliveira MLS, Wollenschlager M, Mark T, Pérez-Lopez R, Macias F. 2011. Leaching of potential hazardous elements of coal cleaning rejects. Environ. Monit. Assess., 175: 109-126.

Sundaray SK, Nayak BB, Lin S, Bhatta D. 2011. Geochemical speciation and risk assessment of heavy metals in the river estuarine sediments - A case study: Mahanadi basin, India. J. Hazard. Mater., 186: 1837-1846.

Zheng N, Liu J, Wang Q, Liang Z. 2010. Heavy metals exposure of children from stairway and sidewalk dust in the smelting district, northeast of China. Atm. Environ., 44: 3239-3245. 